\title{
INFRARED EXCESSES AND MASS LOSS: IMPLICATIONS FOR OB STARS IN CARINA
} AND THE LMC

\author{
A. R. Hyland \\ Mount Stromlo and Siding Spring Observatories, \\ The Australian National University, \\ Canberra, Australia
}

Abstract: A method of deriving mass loss rates using near IR excesses is established and applied to OB stars in Carina and the LMC.

\section{IR OBSERVATIONS AS A DIAGNOSTIC TOOL FOR MASS LOSS}

Methods to derive precise values of the radiation pressure driven mass loss rate are crucial to the development of a broader understanding of the consequences of mass loss from hot luminous stars. One of the most successful methods appears to be the analysis of IR excesses by Barlow and Cohen (1977), (hereinafter referred to as BC). For reasons of observational difficulty all previous analyses have dealt with bright stars. Wright and Barlow (1975) developed a theory for predicting the IR and radio fluxes from a uniform velocity ionized mass loss outflow. Freefree emission from the stellar wind produces an IR excess, the wavelength dependence of which depends upon the rate of mass loss and the form of the velocity law. For a uniform velocity outflow $S_{\nu} \propto v^{2 / 3}$ while for an accelerating flow $B C$ showed that the exponent of $\nu$ is greater than $2 / 3$. In $B C^{\prime}$ 's extensive study of mass loss from 0 stars and $B$ supergiants using observations of $I R$ excesses in the 3-10 $\mu \mathrm{m}$ region, they found that an accelerating flow is in better accord with the data for P Cyg, and applied such a law to derive mass loss rates for their program stars. Surprisingly they found excesses at $3.6 \mu \mathrm{m}$ to be small or zero. Predicted excesses for 3.6-10 $\mu \mathrm{m}$ and 1.25-2.2 $\mu \mathrm{m}$ colors under two assumptions for the flux distribution in the stellar wind are shown in Figure 1 and indicate that a one to one correlation should exist between them. This is confirmed by comparison of the 1.25-2.2 $\mu \mathrm{m}$ excess (which we denote as $E_{f}(J-K)$ ) with $B C^{\prime} s 10 \mu m$ excesses. Thus, values of $\dot{M}$ should be obtainable from observations of $E_{f}(J-K)$, and this method has several advantages over longer wavelength measurements e.g., (a) the magnitudes and excesses can be derived more precisely, (b) much fainter objects can be measured, and (c) the 1.25-2.2 $\mu \mathrm{m}$ region is less affected by emission from cool dust. 


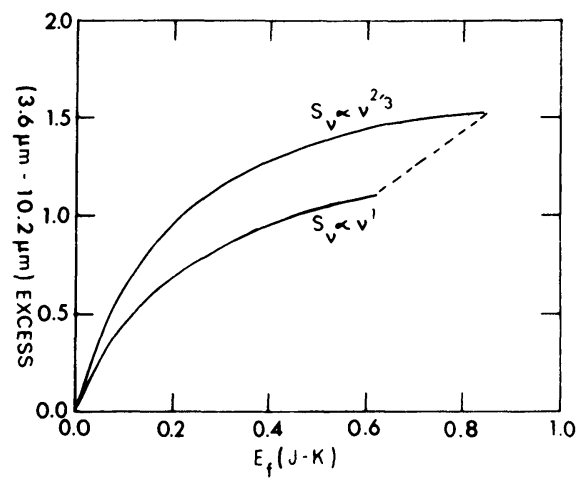

Figure 1. Predicted excesses

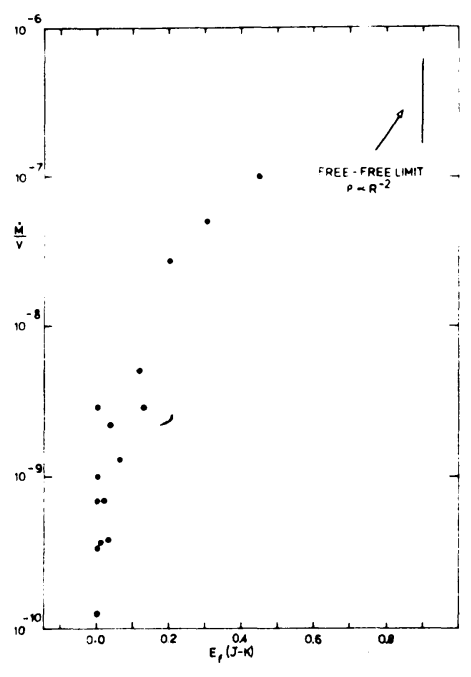

Figure 2. Empirical calibration of $\mathrm{E}_{\mathrm{f}}(\mathrm{J}-\mathrm{K})$ against $\mathrm{M} / \mathrm{v}_{\infty} \mathrm{M}_{\odot} \mathrm{yr}^{-1} /(\mathrm{km} / \mathrm{sec})$.

\section{CALIBRATION OF $\mathrm{E}_{\mathrm{f}}(\mathrm{J}-\mathrm{K})$ IN TERMS OF MASS LOSS}

We have calibrated $E_{f}(J-K)$ in terms of $M / v_{\infty}$ using the results of $B C$. Values of $E_{f}(J-K)$ were determined from published colors (see Schultz and Wiemer 1975, and references therein) using the normal van der Hulst reddening curve and intrinsic $(B-V)$ colors (Johnson 1966). This procedure is prone to an uncertainty $\sim 0.03$, due to the fact that the intrinsic colors derived by Johnson include any component due to freefree emission in the colors of normal stars, our values being too small by this amount. This empirical calibration is shown in Figure 2. Although for $E_{f}(J-K)<0.10$ the result provides little quantitative information, for $E_{f}(J-K) \geqslant 0.10$ the method holds promise for clear estimates of mass loss rates for OB stars.

\section{RESULTS AND DISCUSSION}

Infrared $\mathrm{J}, \mathrm{H}, \mathrm{K}$ magnitudes have been obtained for a series of $O B$ and WR stars in Carina, the LMC, and in interstellar bubbles in the LMC. The observations were made with the Mount Stromlo IR photometer attached to the $3.9 \mathrm{~m}$ AAT and the $1 \mathrm{~m}$ telescope at Siding Spring Observatory. The results are given in Figure 3, from which these points can be made:

(a) The Carina 0 stars (HD93250, 93204, 93205, F100 and F104) (Feinstein, Marraco and Muzzio 1973) have excesses typical of the calibration 0 stars, where $0.05<\mathrm{E}_{\mathrm{f}}(\mathrm{J}-\mathrm{K})>0.12$, depending upon the value of $\mathrm{R}$.

(b) The excesses of the LMC OB emission line stars range up to the limit predicted for radiation pressure induced mass loss. Contamination of 


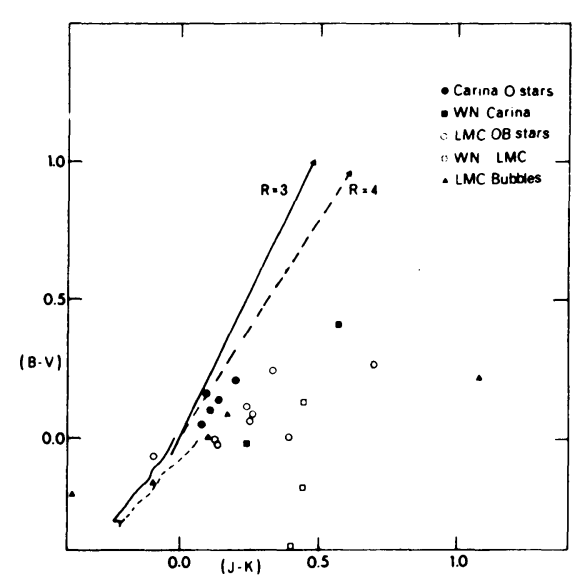

Figure 3. Observations of $O B$ stars in the (B-V) vs. ( $J-K)$ plane. Loci of the intrinsic colors of main sequence and supergiant OB stars are shown as solid and dashed lines respectively. Arrowed lines are reddening lines.

the colors by dust emission was excluded by consideration of the fluxes from $1.25-3.6 \mu \mathrm{m}$.

(c) The Carina WN stars (HD93162 and HD93131) and those in the LMC (Hyland, Thomas and Robinson 1978) have $\mathrm{E}_{\mathrm{f}}(\mathrm{J}-\mathrm{K}) \approx 0.50$, implying extreme rates of mass loss. The LMC stars appear more extreme, but their (B-V) colors may be contaminated by surrounding nebulosity.

(d) The establishment of a method for obtaining mass loss rates of the faint central stars in interstellar bubbles in the LMC was the prime motivation for this study. Our aim was to relate these rates with those inferred from $\mathrm{H} \alpha$ intensities and dynamical measurements of the bubbles. Observations were obtained for stars in the bubbles $\mathrm{N} 70, \mathrm{~N} 9, \mathrm{~N} 185$, and N186 (two stars). Apart from N186 $\|_{1} 1$, whose extreme excess is due to its composite nature, all other bubble stars have small excesses. The central star of the best studied bubble, $N 70$, lies to the extreme left of Figure 3 and has an unexplained IR deficiency. Hence the observed mass loss rates are insufficient to produce and support the bubbles, and continuous mass loss is unacceptable as a mechanism for their formation. An intermittent or eruptive process which produces high mass loss for short periods of time, appears to be required.

\section{RE FE RENCES}

Barlow, M.J., and Cohen, M.: 1977, Astrophys.J., 213,pp. 737-755.

Feinstein, A., Marraco, H.G., and Muzzio, J.C.: 1973, Astron. Astrophys. Supplement, $12, \mathrm{pp} .331-350$.

Hyland, A.R., Thomas, J.A., and Robinson, G.: 1978, Astron.J., 83,pp. 20-25. Johnson, H.L.: 1966, Ann. Rev. Astron. Astrophys., 4,pp. 193-206.

Schultz, G.V., and Wiemer, W.: 1975, Astron.Astrophys.,43,pp. 133-139.

Wright, A.E., and Barlow, M.J.: 1975, Mon.Not.R.astr.Soc., 170,pp.41-51. 


\section{DISCUSSION FOLLOWING HYLAND}

Conti: If one has trouble interpreting the nebular shell as coming from the present star it might be that a previous "event" caused the ejection. The central star now may have a completely different spectrum than previous to this episode.

Hyland: That is certainly true, and the existence of such events may have some bearing on the unusual colors of the central star in $N 70$. Unfortunately, the hypothesis of continuing steady mass outflow as the cause of the "bubble" appears to be untenable.

Cowley: A number of the early OB supergiants in the LMC, which show "normal" blue spectra, in fact turn out to be compcsites in a fainter red supergiant. Can you distinguish these from other kinds of IR excesses?

Hyland: Yes. The three common types of infrared excesses (i.e., the presence of a companion, free-free emission or dust emission) are easily distinguished in a $(\mathrm{J}-\mathrm{H})$ vs. (H-K) color-color diagram. Several emission line $O B$ stars in the LMC have been identified as being composite on the basis of their positions in such a diagram.

Underhill: Could you remind us of the wavelengths of HJK?

Hyland: $\mathrm{H}$ is $1.65 \mu \mathrm{m}, \mathrm{J}$ is $1.25 \mu \mathrm{m}$ and $\mathrm{K}$ is $2.20 \mu \mathrm{m}$. 